\title{
Impact of Physical Activity in Cardiovascular and Musculoskeletal Health: Can Motion Be Medicine?
}

\author{
Gannon L. Curtis ${ }^{\mathrm{a}}$, Morad Chughtai ${ }^{\mathrm{a}}$, Anton Khlopas ${ }^{\mathrm{a}}$, Jared M. Newman ${ }^{\mathrm{a}}$, Rafay Khan ${ }^{\mathrm{b}}$, \\ Shervin Shaffiy ${ }^{\mathrm{c}}$, Ali Nadhim ${ }^{\mathrm{b}}$, Anil Bhave ${ }^{\mathrm{d}}$, Michael A. Mont ${ }^{\mathrm{a}, \mathrm{e}}$
}

\begin{abstract}
Physical activity is a well-known therapeutic tool for various types of medical conditions, including vasculopathic diseases such as coronary artery disease, stroke, type 2 diabetes, and obesity. Additionally, increased physical activity has been proposed as a therapy to improve musculoskeletal health; however, there are conflicting reports about physical activity potentially leading to degenerative musculoskeletal disease, especially osteoarthritis (OA). Additionally, although physical activity is known to have its benefits, it is unclear as to what amount of physical activity is the most advantageous. Too much, as well as not enough exercise can have negative consequences. This could impact how physicians advise their patients about exercise intensity. Multiple studies have evaluated the effect of physical activity on various aspects of health. However, there is a paucity of systematic studies which review cardiovascular and musculoskeletal health as outcomes. Therefore, the purpose of this review was to assess how physical activity impacts these aspects of health. Specifically, we evaluated the effect of various levels of physical activity on: 1) cardiovascular and 2) musculoskeletal health. The review revealed that physical activity may decrease cardiovascular disease and improve OA symptoms, and therefore, motion can be considered a "medicine". However, because heavy activity can potentially lead to increased OA risk, physicians should advise their patients that excessive activity can also potentially impact their health negatively, and should be done in moderation, until further study.
\end{abstract}

Keywords: Physical activity; Cardiovascular health; Musculoskeletal health

\section{Introduction}

Physical activity is a well-known therapeutic tool for various

Manuscript accepted for publication March 14, 2017

${ }^{a}$ Department of Orthopaedic Surgery, Cleveland Clinic, Cleveland, OH, USA bRaritan Bay Medical Center, Perth Amboy, NJ, USA

${ }^{\mathrm{c} S t}$. Georges University School of Medicine, True Blue, Grenada, West Indies ${ }^{\mathrm{d}}$ Sinai Hospital of Baltimore, Baltimore, MD, USA

${ }^{\mathrm{e} C o r r e s p o n d i n g ~ A u t h o r: ~ M i c h a e l ~ A . ~ M o n t, ~ D e p a r t m e n t ~ o f ~ O r t h o p a e d i c s, ~}$ Cleveland Clinic, 9500 Euclid Ave. A40, Cleveland, Ohio 44195, USA.

Email: montm@ccf.org and rhondamont@aol.com

doi: https://doi.org/10.14740/jocmr3001w types of medical conditions [1]. Regular exercise has been shown to have protective effects and benefits in vasculopathic diseases such as coronary artery disease, stroke, type 2 diabetes, and obesity [2]. As the United States population grows older, diseases, such as coronary artery disease and osteoarthritis (OA), will increase in prevalence [3]. Furthermore, aging coincides with physical frailty and functional limitations, which can pose a marked burden on families and healthcare systems $[4,5]$. This has brought a demand to improve the aging population's health and quality of life. Additionally, increased physical activity has been proposed as a therapy to improve musculoskeletal health; however, there are conflicting reports about physical activity potentially leading to degenerative musculoskeletal disease, especially OA [6].

Although physical activity is known to have its benefits, it is unclear as to what amount of physical activity is the most advantageous. Currently, the American Academy of Orthopaedic Surgeons guidelines recommend (strong recommendation) that patients who have symptomatic knee OA engage in quadriceps strengthening and low impact aerobic exercise [7]. However, too much, as well as not enough exercise can have negative consequences, as extensive physical activity has been shown to increase the risk of diseases, such as OA, compared to lower activity levels [8]. This could impact how physicians advise their patients with regard to exercise intensity. Although multiple studies have been conducted to evaluate the effect of physical activity on various aspects of health, there is a paucity of systematic studies which review effects of cardiovascular and musculoskeletal health as outcomes.

It is important to understand how we can maximize the benefits and minimize the risks associated with physical activity. Additionally, it is imperative to analyze the level of physical activity to understand the appropriate level that is beneficial. Therefore, the purpose of this review was to assess how physical activity impacts health. Specifically, we evaluated the effect of various levels of physical activity on: 1) cardiovascular disease and mortality; 2) the development of musculoskeletal disease (OA); and 3) patients who have baseline OA.

\section{Methods}

A pair of literature searches were conducted to identify clinical studies that assessed how physical activity levels affect cardiovascular and musculoskeletal health. We utilized the following search engines: PubMed, EBSCOhost, Ovid, and Embase. 
Studies published between January 1996 and June 2016 were evaluated. The following inclusion criteria were applied: fulltext reports; randomized crossover studies; randomized controlled trials (RCTs); and studies that had cardiovascular or musculoskeletal health components as an outcome. Exclusion criteria included: studies in which the outcome measures did not include cardiovascular or musculoskeletal health; reports published in languages other than English; and non-trial papers (i.e., expert opinions, reviews).

The first literature search performed was aimed to assess the relationship between physical activity level and cardiovascular health. Reports were identified by using a combination of the following search terms: heart diseases/epidemiology, heart diseases/etiology, heart diseases/prevention and control, exercise, and physical fitness. We initially identified 197 reports. These abstracts were reviewed, along with related citations from references, and 188 articles were excluded for various reasons (not in English, case reports, not related, review articles).

The literature search regarding physical activity level effects on musculoskeletal health was performed using the same methods as performed for cardiovascular health. Abstracts were identified using a combination of the following search terms: osteoarthritis/epidemiology, osteoarthritis/etiology, osteoarthritis/prevention and control, exercise, physical fitness. We initially identified 62 reports. These abstracts were reviewed, along with related citations from references, and 52 articles were excluded for various reasons (not in English, case reports, not related, review articles).

When multiple reports of the same patient population were published by the same author, the study with the larger group of patients and/or the longer follow-up was included in the analysis. The opinion of a second author was sought when a consensus decision could not be reached. This resulted in 19 studies for evaluation (nine for cardiovascular and 10 for OA) for analysis.

\section{Results}

\section{Physical activity and cardiovascular health}

Several studies correlated leisure time physical activity levels to incidence of cardiovascular disease and/or mortality (Table 1) [9-17]. Three of nine of these studies analyzed their findings based on gender, and one of nine studies described their findings based on race. The remaining five studies evaluated their entire cohort without focusing on any particular stratifications.

A prospective cohort of 83,034 Japanese citizens were followed by Inoue et al [9] for a mean of 8.7 years in order to establish a relationship between physical activity levels and all-cause mortality. Subjects were asked about the amount of time they performed heavy physical work (none, $<1 \mathrm{~h}, \geq 1 \mathrm{~h}$ ), were sedentary $(<3 \mathrm{~h}, 3-8 \mathrm{~h}, \geq 8 \mathrm{~h})$, or stood and walked $(<1$ $\mathrm{h}, 1-3 \mathrm{~h}, \geq 3 \mathrm{~h}$ ). The data were then transferred into metabolic equivalent (MET) scores with four groupings: lowest, second, third, and highest MET h/day. For men and women, there was an inverse relationship between heart disease risk and MET h/ day (men $\mathrm{P}_{\text {trend }}<0.001$; women $\mathrm{P}_{\text {trend }}=0.001$ ). Patel et al [10] prospectively followed a cohort of 123,216 participants for an average of 13.1 years to examine time spent sitting versus being physically active and relate it to mortality. Patients were asked how much time they spent sitting and how many hours per week they are doing various physical activities. They observed that there was an increased risk of cardiovascular mortality with higher rates of sitting reported (men $\mathrm{P}_{\text {trend }}=0.0007$; women $\mathrm{P}_{\text {trend }}<0.0001$ ), and there was a decrease in cardiovascular mortality with higher levels of physical activity (men $P_{\text {trend }}=0.001$; women $P_{\text {trend }}<0.0001$ ). Kim et al [11] looked at a prospective group of 134,596 subjects who were followed for an average of 13.7 years to assess how time spent sitting effects mortality. Interestingly, the authors found that total time spent sitting per day did not correlate with mortality in men, but women who sat more than 10 total hours per day have an increased risk of cardiovascular mortality (hazard ratio (HR): 1.19 ; P < 0.01). Furthermore, a sub-category of participants who sit and watch at least $5 \mathrm{~h}$ of television per day were found to be at a higher risk of cardiovascular mortality $(\mathrm{P}<0.01)$.

Matthews et al [12] designed a prospective study to identify the effects that physical activity and a sedentary lifestyle have on mortality in black adults compared to white adults. A total of 63,308 participants were recruited, filled out a questionnaire on physical activity levels and were then followed over a 6.4-year period. The authors found a meaningful reduction in cardiovascular mortality in black (HR: $0.81 ; 95 \%$ CI: $0.67-0.98$ ) and white (HR: 0.69; 95\% CI: 0.49 - 0.99) adults who were in the highest activity level. In contrast, blacks who were considered the most sedentary ( $>12 \mathrm{~h} /$ day) did not show an increased risk in cardiovascular disease, whereas white men who were in that same group did have an increase in cardiovascular mortality (HR: 2.18; 95\% CI: 1.34 - 3.54), suggesting that this may vary among different races.

Additionally, Matthews et al [13] performed a prospective study with 240,819 participants who were followed for a mean of 8.5 years. The authors wanted to correlate time spent sitting or watching television with overall mortality, and adjusted for amounts of moderate-heavy physical activity per week. Those who reported the most sedentary hours per day $(\geq 7 \mathrm{~h}$ ) had a greater risk of dying from cardiovascular disease (HR: 1.85 ; 95\% CI: $1.56-2.21$ ) as compared to those who reported the less sedentary hours per day $(<1 \mathrm{~h})$. The analysis also revealed that when highly active patients (i.e., high levels of moderate-to-vigorous physical activity more than $7 \mathrm{~h} /$ week) sit and watch television for a minimum of $7 \mathrm{~h} /$ day, there was an associated increased risk of cardiovascular mortality compared to similar patients who sit and watch for less than $1 \mathrm{~h} /$ day (HR: 2.00; 95\% CI: $1.33-3.00$ )., which is similar to the findings in the Patel et al [10] study. Katzmarzyk [14] followed 16,586 subjects in a prospective study that looked at how long Canadian adults ( $\geq 18$ years old) stand throughout the day and the effect that this has on mortality. The subjects were placed into four groups based on self-reported answers: no standing, standing one-fourth of the day, half of the day and most of the day. There was a significant trend between decreases in cardiovascular mortality with increased time standing per day $\left(\mathrm{P}_{\text {trend }}=0.02\right)$. Furthermore, A Danish study conducted by 


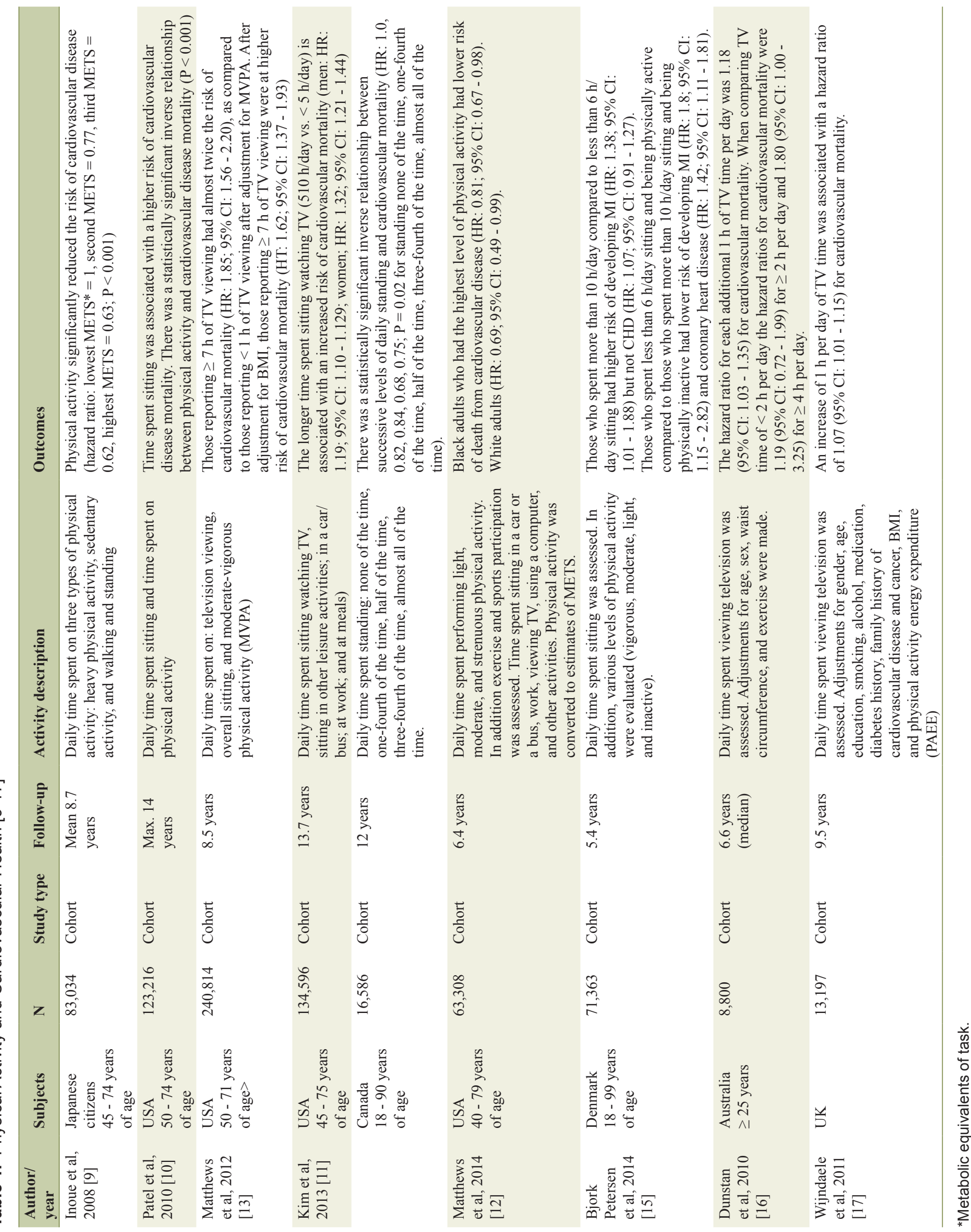


Bjork Petersen et al [15] looked at 71,363 participants to find if coronary heart disease (CHD) and myocardial infarctions (MI) rates were effected by the amount of time spent sitting. The authors showed that sitting more throughout the day ( $\geq$ $10 \mathrm{~h}$ versus $<6 \mathrm{~h}$ ) leads to a significant increase in risk for MI (HR: $1.38 ; \mathrm{P}=0.05$ ), but the relationship to CHD is not defined (HR: $1.07 ; \mathrm{P}=0.59$ ). They also indicated that participants who sat more and were less active had higher rates of MI (HR: 1.8; 95\% CI: 1.15 - 2.82) and CHD (HR: $1.42 ; 95 \%$ CI: 1.11 - 1.81) compared to those who sat less and were more active. Dunstan et al [16] conducted a prospective study in Australia that followed 8,800 individuals for an average of 6.6 years to assess the effect that television viewing time had on mortality. The authors found that individuals who watched more than $4 \mathrm{~h}$ of television per day had higher risks of cardiovascular disease (HR: 1.8; 95\% CI: 1.00 - 3.25) compared to individuals who watched less than $2 \mathrm{~h}$ /day. They also calculated a cardiovascular disease HR of 1.18 (95\% CI: 1.03 - 1.35) for every $1 \mathrm{~h}$ increment in television viewing per day. Similarly, a prospective cohort of 13,197 subjects were followed by Wijndaele et al [17] for a mean of 9.5 years. This study was designed to determine how television viewing related to mortality rates. These authors established that each $1 \mathrm{~h}$ increase in television viewing time led to increased cardiovascular mortality (HR: $1.07,95 \%$ CI: $1.01-1.15$ ).

In summary, although one of three studies on gender demonstrated a benefit in women only, it appears there is a benefit for both genders. In the study discussing race, there appears to be a minimal effect of race on outcomes. The five reports that did not stratify their findings all demonstrate that increased physical activity and decreased leisure time are associated with improved cardiovascular outcomes.

\section{Physical activity and musculoskeletal health}

Multiple studies assessed the relationship between physical activity level and the development of OA (Table 2) [18-27]. There were four studies that described OA as a primary outcome and six that described patients who had OA at baseline.

\section{OA as a primary outcome}

Of the four studies that described OA as a primary outcome, one described only symptomatic OA (SOA), and three described both symptomatic and radiographic OA (ROA). Plotnikoff et al [18] performed a cross-sectional study of 1,808 participants in which they collected self-reports of OA and activity levels followed by an in-person clinical assessment for OA. The study showed that subjects who report standing or walking throughout their day have a decrease in hip OA (odds ratio (OR): $0.5 ; \mathrm{P}=0.038$ ) as well as those who carry/lift light loads throughout their day (OR: $0.45 ; \mathrm{P}=0.037)$. All activity levels did not significantly increase or decrease the risk of knee $\mathrm{OA}$, and heavy workloads did not significantly increase or decrease the risk of hip OA. Obesity (body mass index (BMI) $\geq$ 30) was significantly correlated to an increase in knee (OR: 4.37; $\mathrm{P}<0.001$ ) and hip (OR: 2.52; $\mathrm{P}=0.018$ ) OA.
Felson et al [19] conducted a prospective study of 1,279 patients to determine the long-term outcomes that exercise has on the development of OA. Their study revealed that no type of exercise (walking, jogging, or frequent working up a sweat) was related to an increase or decrease in the risk of developing SOA, ROA or joint space loss. Even the group that reported walking more than 6 miles a week only had an OR of 1.10 when compared to those who did not walk. Furthermore, BMI was assessed and also failed to show a significant correlation to the risk of developing OA. In a retrospective cross-sectional study of 2,637 osteoarthritis initiative (OAI) participants, Lo et al [20] used radiographs, symptom assessments and physical activity surveys to evaluate an association between a history of running and OA. Using an adjusted OR of current runners and previous runners compared to non-runners, they found that the presence of knee pain (OR: 0.76, 0.82; $\mathrm{P}=0.02)$ and SOA (OR: 0.71, 0.88; $\mathrm{P}=0.03$ ) were reduced in subjects with a history of running. There was no significant difference found between the groups in regards to ROA (OR: 0.91, 0.98; $\mathrm{P}=0.5$ ).

A prospective cohort study of 470 patients was performed by McAlindon et al [21] to evaluate the incidence of ROA and SOA related to the intensity and amount of physical activity per day. They found that heavy physical activity led to a significant increase in $\mathrm{ROA}(\mathrm{OR}: 1.3 / \mathrm{h} ; \mathrm{P}=0.006)$. The results for SOA were similar but a small sample prevented a significant outcome.

In summary, it appears that physical activity can lead to improvement in musculoskeletal health, notably, OA. For patients who do not have OA at baseline, all four studies describe some sort of benefit of activity for SOA. However, with regard to ROA, two of the three studies describe physical activity not effecting radiographs, and one study demonstrating heavy physical activity to progressive ROA.

\section{Patients with baseline OA}

There were six studies that described the effects of physical activity in patients who already have a diagnosis of OA at baseline (Table 2). Five of the six studies described symptomatic and functional outcomes, and one study described radiographic outcomes.

Jan et al [22] performed a randomized control study of 98 subjects with knee OA and assessed them for pain, function, walking time and muscle torque after placing them in one of three exercise regimens (high-resistance, low-resistance, and no exercise) for 8 weeks. Both exercise groups improved across all variables after 8 weeks compared to when they began $(\mathrm{P}<$ $0.05)$, and both exercise groups significantly improved across all variables when compared to the control (no exercise) group $(\mathrm{P}<0.008)$. However, there was not a difference between the high- and low-resistance groups.

Similarly, in an RCT conducted by Mangione et al [23], 39 adults with knee OA were allocated into high-intensity and low-intensity stationary cycling groups in order to analyze the effects that this intervention had on function, gait, pain, and aerobic capacity. The authors found that both groups had significant improvements in chair rise time $(\mathrm{P}<0.001)$, 6-min 


\begin{tabular}{|c|c|c|c|c|c|c|c|c|c|}
\hline 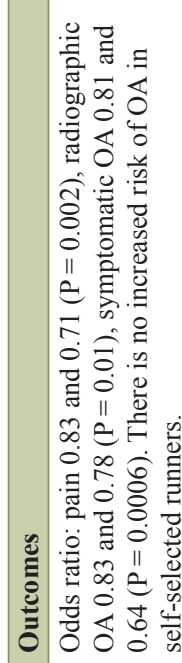 & 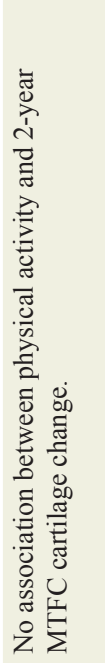 & 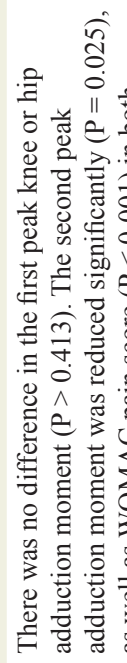 & 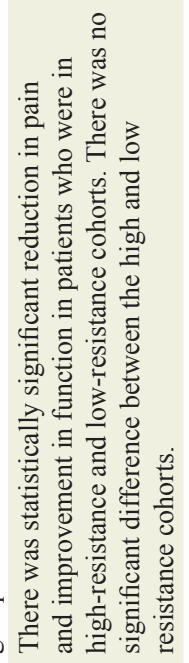 & 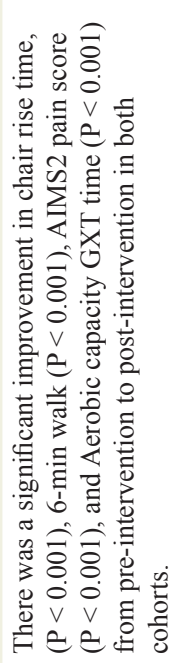 & 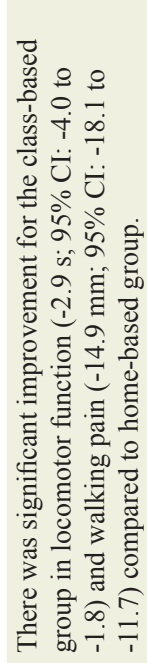 & 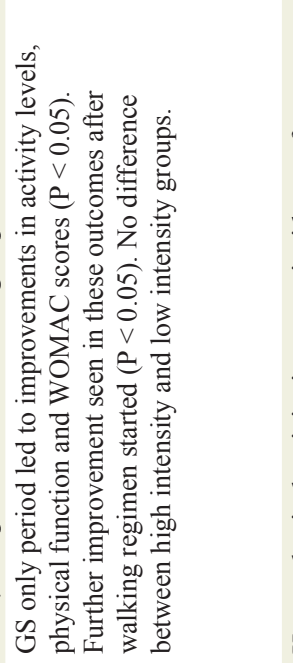 & 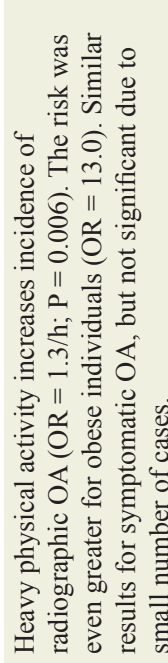 & 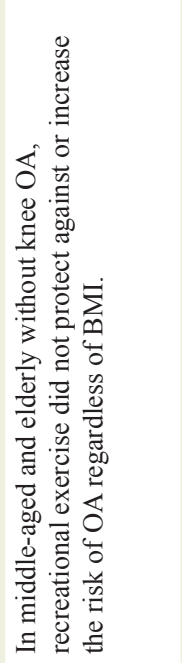 & 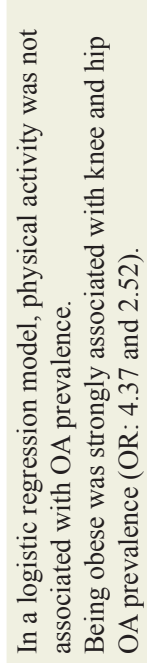 \\
\hline 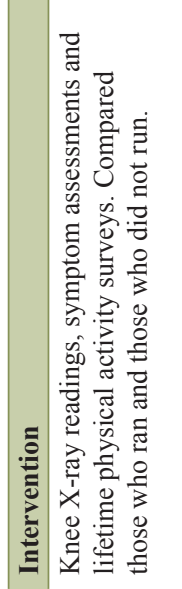 & 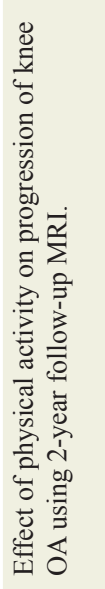 & 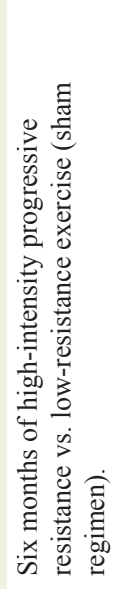 & 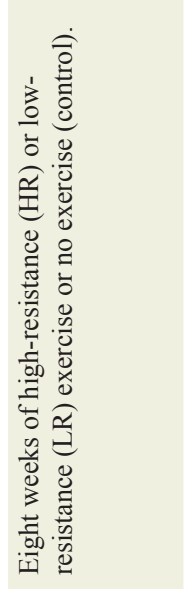 & 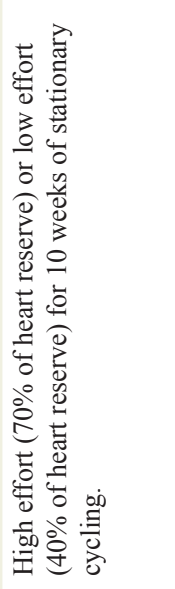 & 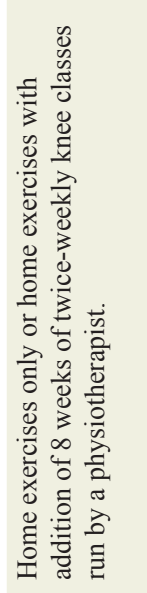 & 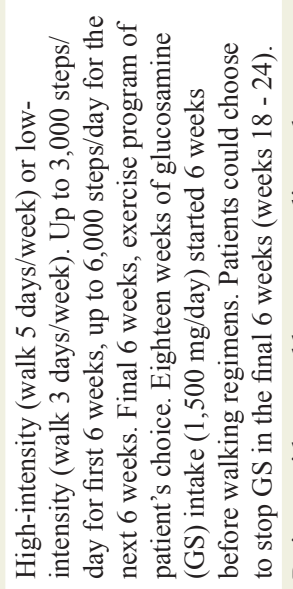 & 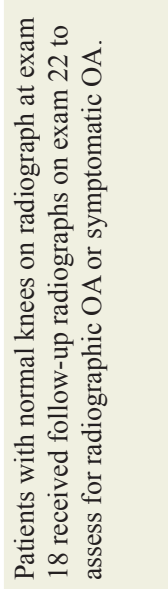 & 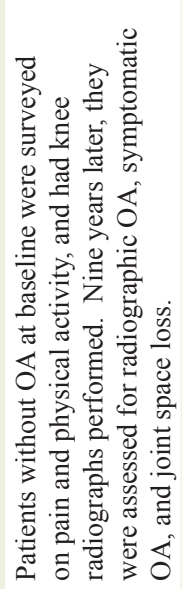 & 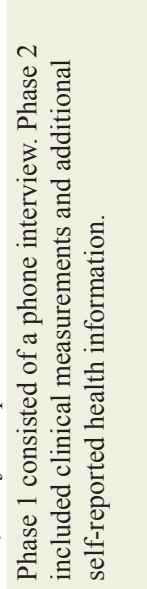 \\
\hline 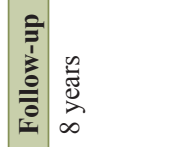 & 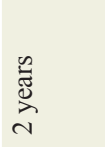 & $\begin{array}{l}\text { n } \\
\text { 言 } \\
\text { ğ }\end{array}$ & $\begin{array}{l}0 \\
\frac{n}{0} \\
0 \\
\infty \\
\infty\end{array}$ & 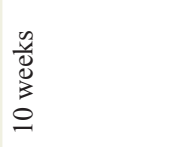 & 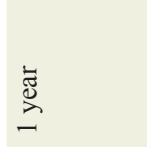 & 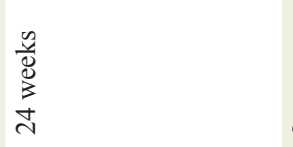 & 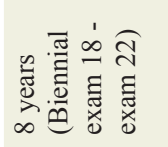 & 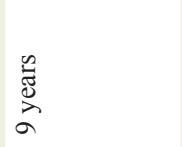 & そ을 \\
\hline 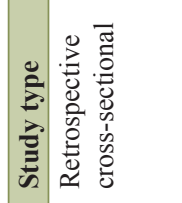 & 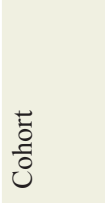 & $\underset{\approx}{5}$ & $\vec{U}$ & 它 & 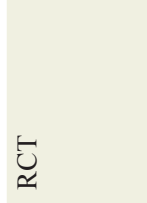 & $\underset{\approx}{5}$ & $\begin{array}{l}\frac{5}{0} \\
\text { o: }\end{array}$ & $\begin{array}{l}\frac{1}{0} \\
\frac{0}{8}\end{array}$ & 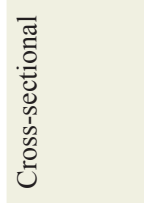 \\
\hline 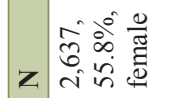 & 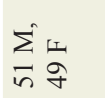 & 崖 & 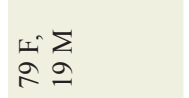 & 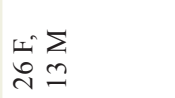 & 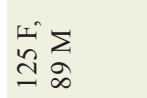 & $\begin{array}{l}\Sigma \Sigma \\
\Sigma\end{array}$ & \& & $\stackrel{\text { సે }}{\stackrel{-}{-}}$ & $\underset{\infty}{\infty}$ \\
\hline 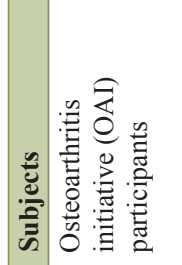 & 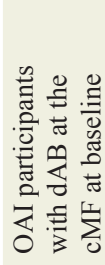 & 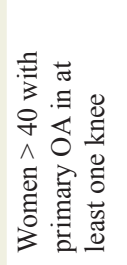 & 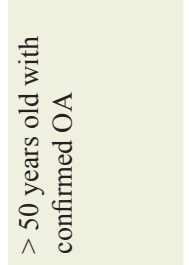 & 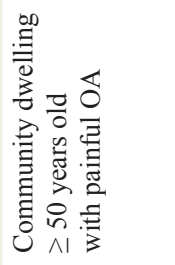 & 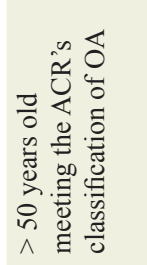 & 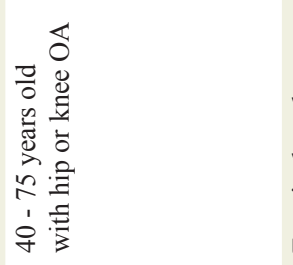 & 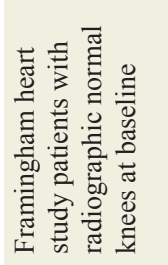 & 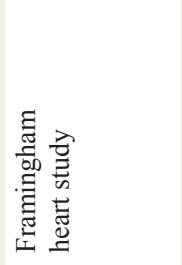 & 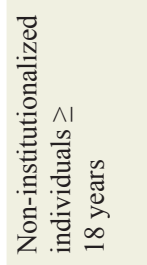 \\
\hline 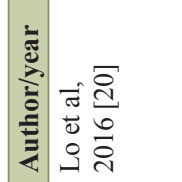 & 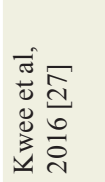 & 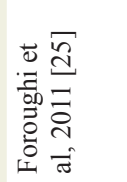 & 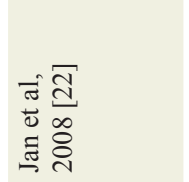 & 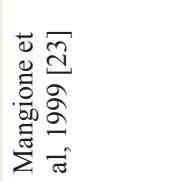 & 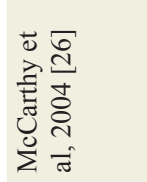 & 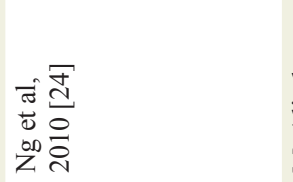 & 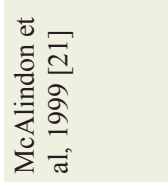 & 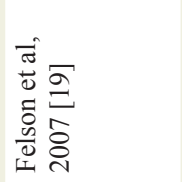 & 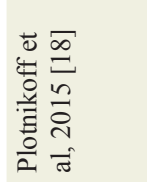 \\
\hline
\end{tabular}


walk test $(\mathrm{P}<0.000)$, pain score $(\mathrm{P}<0.000)$, range of walking speed (slow walking $\mathrm{P}<0.01$; fast walking $\mathrm{P}<0.00$ ), and aerobic capacity $(\mathrm{P}<0.000)$. No meaningful difference was found between the two groups in any outcome.

Similarly, $\mathrm{Ng}$ et al [24] performed an RCT in which 28 subjects with hip or knee OA were placed into a high-intensity (5 day/week) or low-intensity (3 day/week) exercise regimen and all subjects were given glucosamine sulfate (GS). For the first 6 weeks, they only took GS followed by 6 weeks of up to 3,000 steps/day, 6 weeks of up to 6,000 steps/day and finally 6 weeks of an exercise regimen of their choosing. It was the patient's choice to continue taking GS in the final 6 weeks. The results of the study revealed no significant difference between the two groups and the data were combined and analyzed further. After 6 weeks (GS only), there was meaningful improvement in the self-paced step test (SPS) $(\mathrm{P}<0.05)$, stiffness $(\mathrm{P}<$ $0.05)$ and function $(\mathrm{P}<0.05)$. However, the authors note that the subjects increased their physical activity $(\mathrm{P}<0.05)$ when advised not to do so. From week 6 to 24, there was significant gains made in amount of exercise $(\mathrm{P}<0.001)$, SPS $(\mathrm{P}<0.001)$, pain $(\mathrm{P}=0.01)$, and function $(\mathrm{P}<0.001)$, suggesting that the increase in physical activity may have led to improvements in SOA.

A single-blinded RCT was conducted by Furoughi et al [25] to evaluate the effect that lower extremity muscle strengthening has on knee and hip adduction moments in 45 women with confirmed knee OA. The authors found no significant difference between the high-intensity and sham group regarding the primary outcomes of knee and hip adduction $(\mathrm{P}>0.413)$. Both groups had significant reductions in the secondary outcomes of second peak hip adduction moment $(\mathrm{P}=0.025)$ and Western Ontario and McMaster's Universities OA index (WOMAC) pain scores $(\mathrm{P}<0.001)$, suggesting a benefit in patients with SOA. In a similar RCT, McCarthy et al [26] included 214 patients with knee OA, to investigate if a home-based exercise program supplemented with a twice per week physiotherapist led class would affect locomotor activities, pain and disability. The authors found that the group that attended the additional class had significant improvements over the group who did not attend the class. Locomotor function increased 12\% (-2.9 s; $95 \% \mathrm{CI}:-1.8$ to -4.0$)$ and walking pain decreased $27 \%$ (14 $\mathrm{mm}$; 95\% CI: -11.7 to -18.1 ) for the supplemented group. Also, this group experienced meaningful improvements in balance, strength and WOMAC score $(\mathrm{P}<0.05)$.

In a prospective study that included 100 patients with denuded areas of subchondral bone (dABs) at the central weightbearing medial femur (cMF), Kwee et al [27] used an MRI to assess the relationship between physical activity and progression of cartilage loss within the medial tibiofemoral compartment (MTFC) at 2-year follow-up. Using the physical activity scale for the elderly (PASE), there was no significant relationship detected between the physical activity levels and knee cartilage loss. The authors note that the study may have been underpowered to detect slight variations in cartilage loss.

In summary, the five studies describing physical activity in patients who have OA all demonstrated improvements in SOA. The level of physical activity (high versus low intensity), however, did not show differences between one another. There were only differences when either of the activity levels was compared to no activity at all. The one study describing radiographic outcomes did not show progression of OA with activity.

\section{Conclusion}

Increased physical activity level and exercise as well as decreased leisure time, show a consistent benefit in the prevention of cardiovascular disease and mortality [9-15]. An increased amount of time that is spent being sedentary also shows a significant correlation to cardiovascular disease [10, $11,13,15-17]$, even with regular exercise [13]. Overall, time being physically active appears to be the most important influence on the reduction of cardiovascular disease risk. There is a lack of evidence to show if intensity of exercise plays a role.

While one study correlated heavy physical activity with increased rates of ROA [21], three other studies failed to show any association between physical activity levels and OA development [18-20]. Even though running can be viewed as a high-impact exercise, it was not shown to have any impact on the development of OA [20]. Physical activity was shown to reduce symptoms in patients with confirmed OA [22-27].

Physical activity may decrease cardiovascular disease and improve OA symptoms, and therefore, motion can be considered a "medicine". However, because heavy activity can potentially lead to increased OA risk, physicians should advise their patients that excessive activity can also potentially impact their health negatively, and should be done in moderation, until further study, for prevention and treatment of cardiovascular and musculoskeletal diseases. Future studies need to assess this more carefully. Specifically, studies need to better define and standardize different levels of physical activity, and assess their impact on cardiovascular and musculoskeletal health. Cohorts that should be assessed include both patients with and without baseline vasculopathic disease and OA. Additionally, because both cardiovascular and musculoskeletal diseases are tied to obesity, future studies should assess effects of varying levels of weight on these outcomes. This should also be studied in the context of diet, as it is well known to be linked to obesity.

\section{Disclosures}

Dr. Chughtai is a consultant for, or has received institutional or research support from the following companies: Sage Products, Stryker, DJO Global. Dr. Mont is a consultant for, or has received institutional or research support from the following companies: Sage Products, TissueGene, OnGoing Care Solutions, DJO Global, Microport, Orthosensor, National Institutes of Health, Stryker, Medical Compression Systems, Johnson \& Johnson, Pacira Pharmaceuticals, Merz. He is on the editorial/governing board of the American Journal of Orthopedics, Journal of Arthroplasty, Journal of Knee Surgery, and Surgical Technology International. Mr. Bhave is a consultant for, or has received institutional or research support from the following companies: DJO Global. 


\section{References}

1. Sallis R. Exercise is medicine: a call to action for physicians to assess and prescribe exercise. Phys Sportsmed. 2015;43(1):22-26.

2. Lee IM, Shiroma EJ, Lobelo F, Puska P, Blair SN, Katzmarzyk PT. Effect of physical inactivity on major non-communicable diseases worldwide: an analysis of burden of disease and life expectancy. Lancet. 2012;380(9838):219-229.

3. Kanasi E, Ayilavarapu S, Jones J. The aging population: demographics and the biology of aging. Periodontol 2000. 2016;72(1):13-18.

4. Vellas B. [Prevention of frailty and dependency in older adults]. Bull Acad Natl Med. 2013;197(4-5):1009-1017; discussion 1017-1009.

5. Villareal DT, Banks M, Siener C, Sinacore DR, Klein S. Physical frailty and body composition in obese elderly men and women. Obes Res. 2004;12(6):913-920.

6. Kujala UM, Kaprio J, Sarna S. Osteoarthritis of weight bearing joints of lower limbs in former elite male athletes. BMJ. 1994;308(6923):231-234.

7. Treatment of Osteoarthritis of the Knee (2nd edition) n.d. http://www.orthoguidelines.org/guidelinedetail?id=1054 (accessed September 1, 2017).

8. Vingard E, Alfredsson L, Goldie I, Hogstedt C. Sports and osteoarthrosis of the hip. An epidemiologic study. Am J Sports Med. 1993;21(2):195-200.

9. Inoue M, Iso H, Yamamoto S, Kurahashi N, Iwasaki M, Sasazuki S, Tsugane S. Daily total physical activity level and premature death in men and women: results from a large-scale population-based cohort study in Japan (JPHC study). Ann Epidemiol. 2008;18(7):522-530.

10. Patel AV, Bernstein L, Deka A, Feigelson HS, Campbell PT, Gapstur SM, Colditz GA, et al. Leisure time spent sitting in relation to total mortality in a prospective cohort of US adults. Am J Epidemiol. 2010;172(4):419-429.

11. Kim Y, Wilkens LR, Park SY, Goodman MT, Monroe KR, Kolonel LN. Association between various sedentary behaviours and all-cause, cardiovascular disease and cancer mortality: the Multiethnic Cohort Study. Int J Epidemiol. 2013;42(4):1040-1056.

12. Matthews CE, Cohen SS, Fowke JH, Han X, Xiao Q, Buchowski MS, Hargreaves MK, et al. Physical activity, sedentary behavior, and cause-specific mortality in black and white adults in the Southern Community Cohort Study. Am J Epidemiol. 2014;180(4):394-405.

13. Matthews CE, George SM, Moore SC, Bowles HR, Blair A, Park Y, Troiano RP, et al. Amount of time spent in sedentary behaviors and cause-specific mortality in US adults. Am J Clin Nutr. 2012;95(2):437-445.

14. Katzmarzyk PT. Standing and mortality in a prospective cohort of Canadian adults. Med Sci Sports Exerc. 2014;46(5):940-946.

15. Bjork Petersen C, Bauman A, Gronbaek M, Wulff Helge J, Thygesen LC, Tolstrup JS. Total sitting time and risk of myocardial infarction, coronary heart disease and allcause mortality in a prospective cohort of Danish adults.
Int J Behav Nutr Phys Act. 2014;11:13.

16. Dunstan DW, Barr EL, Healy GN, Salmon J, Shaw JE, Balkau B, Magliano DJ, et al. Television viewing time and mortality: the Australian Diabetes, Obesity and Lifestyle Study (AusDiab). Circulation. 2010;121(3):384-391.

17. Wijndaele K, Brage S, Besson H, Khaw KT, Sharp SJ, Luben R, Wareham NJ, et al. Television viewing time independently predicts all-cause and cardiovascular mortality: the EPIC Norfolk study. Int J Epidemiol. 2011;40(1):150-159.

18. Plotnikoff R, Karunamuni N, Lytvyak E, Penfold C, Schopflocher D, Imayama I, Johnson ST, et al. Osteoarthritis prevalence and modifiable factors: a population study. BMC Public Health. 2015;15:1195.

19. Felson DT, Niu J, Clancy M, Sack B, Aliabadi P, Zhang Y. Effect of recreational physical activities on the development of knee osteoarthritis in older adults of different weights: the Framingham Study. Arthritis Rheum. 2007;57(1):6-12.

20. Lo GH, Driban JB, Kriska AM, McAlindon TE, Souza RB, Petersen NJ, Storti KL, et al. Is There an Association Between a History of Running and Symptomatic Knee Osteoarthritis? A Cross-Sectional Study From the Osteoarthritis Initiative. Arthritis Care Res (Hoboken). 2017;69(2):183-191.

21. McAlindon TE, Wilson PW, Aliabadi P, Weissman B, Felson DT. Level of physical activity and the risk of radiographic and symptomatic knee osteoarthritis in the elderly: the Framingham study. Am J Med. 1999;106(2):151-157.

22. Jan MH, Lin JJ, Liau JJ, Lin YF, Lin DH. Investigation of clinical effects of high- and low-resistance training for patients with knee osteoarthritis: a randomized controlled trial. Phys Ther. 2008;88(4):427-436.

23. Mangione KK, McCully K, Gloviak A, Lefebvre I, Hofmann M, Craik R. The effects of high-intensity and low-intensity cycle ergometry in older adults with knee osteoarthritis. J Gerontol A Biol Sci Med Sci. 1999;54(4):M184-190.

24. Ng NT, Heesch KC, Brown WJ. Efficacy of a progressive walking program and glucosamine sulphate supplementation on osteoarthritic symptoms of the hip and knee: a feasibility trial. Arthritis Res Ther. 2010;12(1):R25.

25. Foroughi N, Smith RM, Lange AK, Baker MK, Fiatarone Singh MA, Vanwanseele B. Lower limb muscle strengthening does not change frontal plane moments in women with knee osteoarthritis: A randomized controlled trial. Clin Biomech (Bristol, Avon). 2011;26(2):167-174.

26. McCarthy CJ, Mills PM, Pullen R, Richardson G, Hawkins N, Roberts CR, Silman AJ, et al. Supplementation of a home-based exercise programme with a class-based programme for people with osteoarthritis of the knees: a randomised controlled trial and health economic analysis. Health Technol Assess. 2004;8(46):iii-iv, 1-61.

27. Kwee RM, Wirth W, Hafezi-Nejad N, Zikria BA, Guermazi A, Demehri S. Role of physical activity in cartilage damage progression of subjects with baseline full-thickness cartilage defects in medial tibiofemoral compartment: data from the Osteoarthritis Initiative. Osteoarthritis Cartilage. 2016;24(11):1898-1904. 